\title{
Assessment of DNA Repair Mechanisms to Determine the Susceptibility of Non-small Cell Lung Cancer to Alkylating Agents
}

Ivette F. Emery ${ }^{1,2 *}$, Chiara Battelli1,2, Li Cai ${ }^{3}$ and Daniel M. Hayes ${ }^{1}$

${ }^{1}$ Maine Center for Cancer Medicine, Scarborough, ME 04074

${ }^{2}$ Maine Medical Center, Portland, ME 04102

${ }^{3}$ LabCorp, Center for Molecular Biology \& Pathology, Research Triangle Park, NC

\section{Abstract}

Background: The status of DNA repair systems impacts the sensitivity of tumors to chemotherapeutics. The anti-neoplastic activity of temozolomide, an alkylating agent, is affected by the enzyme $\mathrm{O}^{6}$-Methylguanine-DNA methyltransferase (MGMT), and by the mismatch repair system (MMR). Lack of MGMT makes a tumor susceptible to temozolomide provided MMR is functional. A non-functional MMR renders the tumor resistant to alkylating agents. We assayed MGMT and MMR in order to estimate the proportion of patients affected by NSCLC who may derive benefit from temozolomide, an alkylating agent not currently used in lung cancer.

Method: MGMT and MMR were assayed by promoter methylation testing and by microsatellite instability testing, respectively, on 96 paraffin-embedded NSCLC samples. Methylation testing, which detects gene silencing, was conducted by methylation-specific PCR of the MGMT promoter. MSI testing, which detects a non-functional MMR, was conducted by multiplex PCR of six microsatellite regions.

Results: Ninety three samples yielded interpretable results. Eighty of those $(83 \%)$ had no defects detected in either MGMT or MMR. Nine samples (10\%) had methylation of the MGMT promoter and four samples $(4 \%)$ had microsatellite instability. The defects were not overlapping.

Conclusions: Our results indicate that approximately $10 \%$ of NSCLC may be susceptible to alkylating agents and that in the remaining population, only $4 \%$ will be resistant to these agents. Our understanding of the molecular characteristics of NSCLC suggests that alkylating agents, such as temozolomide, alone or in combination with MGMT inhibitors, may be viable option to treat selected NSCLC patients.

Keywords: Methylguanine-DNA methyltransferase (MGMT); Mismatch Repair (MMR); Temozolomide (TMZ); Promoter methylation; Microsatellite instability (MSI)

\section{Introduction}

Lung cancer continues to be the leading cause of cancer-related deaths in the US, with a 5-year survival rate of only $15.7 \%$ [1]. NSCLC encompasses the largest group within this category and represents an important population for which to target efforts to increase the size of the chemotherapeutic arsenal. Currently, platinum-based chemotherapy results in a statistically significant survival benefit as compared to best supportive care in all stages of NSCLC [2]. However, the various platinum combinations have generated a plateau in overall response and survival benefit.

Temozolomide is an oral alkylating agent that is routinely used in high grade gliomas. The FDA approval of temozolomide followed a clinical trial that showed that the addition of temozolomide improved median survival in patients with glioblastoma multiforme from 12.1 to 14.6 months and improved the 2 -year survival rate from $10.4 \%$ to $26.5 \%$, as compared to radiation therapy alone [3]. An accompanying study showed that the patients that benefited from temozolomide were those that had the MGMT gene silenced by promoter methylation [4]. MGMT is a repair enzyme, which reverses the temozolomide-induced DNA damage and thus, its activity is a mechanism of resistance to this agent [5]. Promoter methylation results in gene silencing and abrogates this resistance mechanism [6-7].

Temozolomide-induced damage leads to cell death via the activity of the MMR system. This system is composed of a family of proteins, orthologs of the prokaryotic MutS and MutL proteins. They assemble at the site of a DNA mismatch, which leads directly to cell cycle arrest and cell death. If the MMR system is disabled, alkylation damage accumulates without triggering cell killing [8]. Acquisition of mutations in the MMR system is another mechanism of resistance to alkylating agents [9].

The functional status of the MMR system and of the MGMT enzyme can be assessed by commercially available molecular tests. MMR deficiency is diagnosed by a high degree of instability in regions of repetitive DNA, a phenotype called microsatellite instability (MSI). $M G M T$ silencing is detected by the presence of methyl groups in the MGMT promoter sequences. We conducted MGMT methylation and MSI tests on paraffin-embedded NSCLC samples, in order to determine the proportion of patients who may derive benefit from temozolomide or other alkylating agents.

\section{Methods}

\section{Samples}

Ninety six (96), histologically confirmed cases of NSCLC were used in this study. Formalin-fixed, paraffin-embedded (FFPE) samples

*Corresponding author: Ivette F. Emery, PhD, Maine Medical Center Cancer Institute, 100 Campus Drive, Suite 102, Scarborough, ME 04074, USA, E-mail: emeryi@mmc.org

Received February 28, 2011; Accepted May 15, 2011; Published May 15, 2011

Citation: Emery IF, Battelli C, Cai L, Hayes DM (2011) Assessment of DNA Repair Mechanisms to Determine the Susceptibility of Non-small Cell Lung Cancer to Alkylating Agents. J Cancer Sci Ther 3: 092-095. doi:10.4172/1948-5956.1000067

Copyright: (C) 2011 Emery IF, et al. This is an open-access article distributed unde the terms of the Creative Commons Attribution License, which permits unrestricted use, distribution, and reproduction in any medium, provided the original author and source are credited. 
were sectioned in 10 micron slices and four serial sections, mounted on glass slides, were used for each test. A single additional section was stained with Hematoxylin and Eosin and used to mark the viable tumor area. The MGMT promoter methylation test was performed at OncoMethylome (now MDxHealth) (Amsterdam, The Netherlands), and the MSI test was performed at Labcorp (Research Triangle Park, NC). This translational study was approved by the Internal Review Board of the Maine Medical Center.

\section{MGMT methylation test}

Detection of promoter methylation of the MGMT gene was conducted as previously published [10]. Briefly, genomic DNA was extracted from sections using the classical phenol/chloroform extraction method. DNA was then subjected to bisulfate treatment using the EZ DNA Modification Kit (ZYmo, Orange, CA). Unmethylated cytosines, but not methylated cytosines, are modified into uracil by this treatment. Methylation-specific polymerase chain reactions (PCR) was performed for the MGMT promoter sequence using specific primers and the Amplifluor assay format on a ABI Prism 7900HT instrument (Applied Biosystems, Foster City, CA). $\beta$-actin was used as a reference gene in the assay using primers outside any CpG islands. The MGMT target sequence is located on chromosome 10 between positions 131155505 and 131155619 , while the $\beta$-actin target sequence is located on chromosome 7 between positions 5538428 and 5538325 (RefSeq, NM_002412). The amplicon sizes for the meth-MGMT and the $\beta$-actin analytes are $136 \mathrm{bp}$ and $125 \mathrm{bp}$, respectively.

The amplicon copy number for MGMT and $\beta$-actin were generated using the SDS 2.2 software (Applied Biosystems, Foster City, CA) as previously described [10]. Briefly, this program determines the cycle number at which the amplification curves cross the threshold value set automatically by the software and then uses it to calculate the copy number based on a linear regression of the values plotted on a standard curve. To compensate for variations in copy number due to sample differences, the derived meth-MGMT copy numbers were divided by the copy number of the control analyte $\beta$-actin for the same sample. The resulting value was multiplied by 1000 to produce a ratio value. Ratio values above 10 are considered methylated samples.

\section{MSI Test}

Detection of microsatellite instability in our samples was conducted as previously described [11]. Briefly, tissue was microdissected from tumor and normal tissue according to the H\&E slide. DNA was isolated using xylene/ethanol deparaffinization followed by Proteinase $\mathrm{K}$ digestion and heat inactivation. Amplification of six microsatellite markers, TGFBRII, BAT26, BAT25, D5S346, D17S250 and D2S123, was accomplished using specific primer sets (LabCorp, RTP, NC) and phosphoramidite fluorescent labels (IDT, IA). Labeled PCR products were detected using the ABI 310 Genetic Analyzer and GeneScan collection software (Applied Biosystems, Foster City, CA). The identification of normal or tumor allele amplicon sizes was accomplished by examining the appropriate electropherogram (normal and tumor) and determining the predominant amplican size(s) for each allele at each locus, i.e. the greatest peak height(s). If the tumor specimen has novel amplicon lengths in relation to the patient's normal amplicon length(s) at a specific locus, that locus was considered positive for MSI. Samples were scored as following: MSI-high included samples were 2 loci or more exhibited microsatellite instability, MSI-low included samples were 1 loci exhibited microsatellite instability and MSI-stable included all samples where no loci exhibited microsatellite instability.

Loss of heterozygosity (LOH) was also determined for the microsatelliate markers. $\mathrm{LOH}$ was defined as complete or partial loss of one of two alleles at a heterozygous locus in tumor-derived DNA compared with normal DNA. Scoring of the alleles was based on comparison of the peak height ratio of a heterozygous matching normal and tumor sample. The peak height ratio was obtained by dividing the height of allele 1 by the height of allele 2 . This was done for both normal and the tumor samples. The $\mathrm{T} / \mathrm{N}$ ratio of the ratios was then calculated as: $[(\mathrm{A} 1 \mathrm{~T} / \mathrm{A} 2 \mathrm{~T}) /(\mathrm{A} 1 \mathrm{~N} / \mathrm{A} 2 \mathrm{~N})]$. $\mathrm{LOH}$ was scored if the $\mathrm{T} / \mathrm{N}$ ratio deviates more that $30 \%$ from the reference value of 1 .

\section{Results}

We determined the MGMT promoter methylation status and the microsatellite instability of one hundred and eight (108) tumor tissue samples from 96 patients that were treated at the Maine Center for Cancer Medicine for a diagnosis with Non-small cell lung cancer. The characteristics of this patient population are noted on Table 1. Eighty (80) of those patients had methylation levels below 10 ("unmethylated"), 9 patients had above-threshold methylation levels ("methylated"), 5 patients had methylation levels above 10 but below threshhold ("unmethylated"), and 3 patients had an "invalid" result. There were 11 patients with 2 or more tissue blocks. Results of those duplicate samples were all concordant with one exception; two samples from the same patient were found, in one case to have unmethylated promoter and in the other case to have an "invalid" result. The overall rate of methylated MGMT promoter among our NSCLC patients was $9.6 \%$, indicating that this cancer type is not especially susceptible to temozolomide (Table 2).

Ninety one (91) of the patients had tumors that displayed microsatellite stability (MSIS), 2 patients had a low level of microsatellite instability (MSIL), 2 patients had a high level of microsatellite instability (MSIH), and 1 patient had no normal tissue for comparison purposes and therefore had an invalid result. There were 11 patients with 2 or more tissue blocks. Results of those duplicate samples were concordant except in two cases. In both those cases, one sample showed some degree of instability and the other had a stable result. The overall rate of microsatellite instability among our NSCLC patients was 3.7\% indicating that this cancer type is unlikely to be resistant to alkylating agents. There were no patients with both MGMT promoter methylation and microsatellite instability (Table 2 ).

\begin{tabular}{|l|l|}
\hline & No \\
\hline TOTAL & 96 \\
\hline Gender & \\
\hline Female & 48 \\
\hline Male & 48 \\
\hline Age & \\
\hline Avg. age & 65.7 \\
\hline Range & $42-88$ \\
\hline Smoking history & \\
\hline Smokers & 72 \\
\hline Non-smokers & 2 \\
\hline Unknown & 22 \\
\hline Avg. pack-years of smokers & 48.3 \\
\hline Histology & \\
\hline Adenocarcinoma & 53 \\
\hline Squamous & 20 \\
\hline Bronchioalveolar & 6 \\
\hline Large cell & 3 \\
\hline Mixed & 2 \\
\hline NSC not further specified & 12 \\
\hline
\end{tabular}

Table 1: Patient characteristics. 


\begin{tabular}{|c|c|c|c|c|c|c|c|c|c|c|c|}
\hline \multirow[b]{2}{*}{ Sample } & \multirow[b]{2}{*}{ Tissue } & \multicolumn{3}{|c|}{ MGMT Promoter Methylation Test } & \multicolumn{7}{|c|}{ Microsatellite Instability Test } \\
\hline & & MGMT & $\beta$-Actin & Result & TGFBRII & D5S346 & BAT26 & BAT25 & D17S250 & D2S123 & Result \\
\hline LNG3 & IB adeno & 407 & 6654 & meth & Neg & Neg & Neg & Neg & Neg & Neg & MSIS \\
\hline LNG39 & IA adeno & 18 & 1255 & meth & Neg & Neg & Neg & Neg & Neg & Neg & MSIS \\
\hline LNG66 & IV adeno-br met & 52 & 6014 & meth & Neg & Neg & Neg & Neg & Neg & Neg & MSIS \\
\hline LNG68 & IV adeno-lung & 661 & 9421 & meth & Neg & Neg & Neg & Neg & Neg & Neg & MSIS \\
\hline LNG72 & IIIB squam & 56 & 2935 & meth & Neg & $\mathrm{LOH}$ & Neg & Neg & Neg & Neg & MSIS \\
\hline LNG75 & IB adeno & 85 & 4716 & meth & Neg & Neg & Neg & Neg & Neg & Neg & MSIS \\
\hline LNG79 & IV adeno-In & 56 & 3526 & meth & Neg & Neg & Neg & Neg & Neg & Neg & MSIS \\
\hline LNG97 & IIA squam & 281 & 29547 & meth & Neg & $\mathrm{LOH}$ & Neg & $\mathrm{Neg}$ & Neg & Neg & MSIS \\
\hline LNG109 & IB NSCLC & 897 & 5431 & meth & Neg & Neg & Neg & Neg & Neg & Neg & MSIS \\
\hline LNG40 & IIIB large cell & $<10$ & 1994 & unmeth & Neg & Neg & Neg & MSI & Neg & Neg & MSIL \\
\hline LNG48 & IIB large cell & $<10$ & 6607 & unmeth & Neg & MSI & $\mathrm{Neg}$ & $\mathrm{Neg}$ & Neg & Neg & MSIL \\
\hline LNG63 & IIIA squam & $<10$ & 781 & unmeth & Neg & MSI & Neg & Neg & MSI & Neg & MSIH \\
\hline LNG67 & IA adeno & $<10$ & 4971 & unmeth & $\mathrm{Neg}$ & $\mathrm{Neg}$ & MSI & $\mathrm{Neg}$ & MSI & $\mathrm{Neg}$ & MSIH \\
\hline
\end{tabular}

Table 2: MGMT promoter methylation and MSI test results. The results of the MGMT promoter methylation test and the Microsatellite instability test are shown for the 13 tumor samples that displayed defects in either of these two repair mechanisms. The first column indicates the patient sample ID and the second column indicates the tumor stage and histology of each sample. The copy number of the MGMT promoter amplicon is shown on the third column, while the copy number of the reference, $\beta$-Actin, amplicon in shown on the fourth column. The fifth column indicates whether the sample was categorized as having the MGMT promoter region methylated or unmethylated. Columns 6-11 show the instability of 6 microsatellite regions. Negative (Neg) indicates stable regions, MSI indicates regions that displayed instability, and LOH indicates regions with loss of heterozygosity. The last column indicates whether a sample was categorized as MSI-stable (MSIS) or samples with no regions of instability, MSI-high (MSIH) or samples were 2 loci or more exhibited microsatellite instability, or MSI-low(MSIL) or samples were 1 loci exhibited microsatellite instability

There were no detectable associations between the status of these repair mechanisms and smoking history, histology or tumor grade. Also, we did not discern any remarkable differences in the response or survival between the patients that had aberrations in either MGMT or MSI and those that did not. The average progression free survival among patients with MGMT promoter methylation was 21 months, and the median was 13 months. For those with MSI, the average progression free survival was 17 months while the median was 16 month. For the reminder of the NSCLC population, the average progression free survival was 16 month and the median was 11 months. The small number of NSCLC patients with MGMT promoter methylation or a positive MSI test precluded obtaining statistically significant differences.

\section{Discussion}

The disabling of repair mechanisms occurs as part of the process of cancer progression. It leads to the so-called "mutator phenotype", which results in the rapid accumulation of mutations and, over time, the selection of tumor clones that are progressively more aggressive and chemoresistant. However, this phenomenon at times opens windows in which the tumor becomes susceptible to specific chemotoxic agents. In the case of alkylating agents such as temozolomide, silencing of the MGMT gene by epigenetic changes in promoter methylation, renders a tumor susceptible to this agent, but only as long as the MMR system remains functional. If MMR becomes disabled, the opportunity to kill tumor cells with this class of agents closes.

Early results indicated that as many as $20 \%$ of lung cancers display MGMT promoter methylation [12]. Later studies have determined that percentage to be lower [13]. Our results indicate that approximately $10 \%$ of NSCL tumors have the MGMT gene silenced by promoter methylation. This is in contrast with the case in glioblastoma multiforme (GBM), where as many as $45 \%$ of tumors display this epigenetic change [4]. For this reason, benefit to temozolomide could be observed in unselected GBM populations.

The proportion of NSCLC cases that display inactivation of MMR was unknown and said to range between 0 to $70 \%$ [14]. Our results indicate that this percentage is very low $(4 \%)$. The determination of this second number is arguably more important than the frequency of MGMT silencing. This is because if MMR is disabled, there are no known manipulations to re-activate it. However, there are inhibitors currently in clinical trials that can lower MGMT expression [15]. In other words, the proportion of NSCLC with a functional MMR system would set the upper limit for the number of patients that may benefit from temozolomide. Indeed the percentage of GBM patients with MMR deficiency is very low [16], in contrast with that in found in the colorectal cancer population where MMR deficiency, either inherited or sporadic, is much more prevalent [17].

Given the dramatic benefit derived from temozolomide in glioma patients with MGMT promoter methylation [3], the results of this translational study argue for the pursuit of early phase clinical trials of temozolomide in MGMT-deficient NSCLC patients. Moreover, if ongoing clinical trials to test the efficacy of MGMT inhibitors prove positive in GBM patients, it would open the door for similar studies in NSCLC. Interestingly, temozolomide has been studied in patients with brain metastasis from NSCLC and other solid tumors [18,19]. Although these studies showed a trend towards improved survival, the results did not reach statistical significance. We could argue that, in the case of NSCLC, this may be due to a low rate of MGMT promoter methylation and the resulting low sensitivity to temozolomide. Two approaches based on our results may lead to improved outcomes: 1) preselection of the $10 \%$ of NSCLC patients with MGMT methylation, 2) coadministration of temozolomide with MGMT inhibitors. Translational studies such as the present study provide background molecular data for more rational clinical trial design.

\section{Acknowledgements}

We would like to thank Kathleen Carrier, histologist at the Maine Medical Center Research Institute, for her expert sectioning of all the tissue blocks and Greg Jones, Program manager at MDxHealth, for mediating the MGMT promoter methylation testing. This work was funded by an unrestricted research grant from Schering-Plough XX-3807 (IFE \& CB). 
Citation: Emery IF, Battelli C, Cai L, Hayes DM (2011) Assessment of DNA Repair Mechanisms to Determine the Susceptibility of Non-small Cell Lung Cancer to Alkylating Agents. J Cancer Sci Ther 3: 092-095. doi:10.4172/1948-5956.1000067

\section{References}

1. http://www.cancer.gov/cancertopics/pdq/treatment/non-small-cell-lung/ HealthProfessional

2. http://www.nccn.org/professionals/physician_gls/PDF/nscl.pdf

3. Stupp R, Mason WP, van den Bent MJ, Weller M, Fisher B, et al. (2005) Radiotherapy plus concomitant and adjuvant temozolomide for glioblastoma. N Engl J Med 352: 987-996.

4. Hegi ME, Diserens AC, Gorlia T, Hamou MF, de Tribolet N, et al. (2005) MGMT gene silencing and benefit from temozolomide in glioblastoma. $\mathrm{N}$ Engl $\mathrm{J}$ Med 352: 997-1003

5. Pegg AE (2000) Repair of O(6)-alkylguanine by alkyltransferases. Mutat Res 462: 83-100.

6. Qian XC, Brent TP (1997) Methylation hot spots in the 5' flanking region denote silencing of the O6-methylguanine-DNA methyltransferase gene. Cancer Res 57: $3672-3677$

7. Paz MF, Yaya-Tur R, Rojas-Marcos I, Reynes G, Pollan M, et al. (2004) CpG island hypermethylation of the DNA repair enzyme methylstransferase predicts response to temozolomide in primary gliomas. Clin Cancer Res 10: 4933-4938.

8. O'Brien V, Brown R (2006) Signalling cell cycle arrest and cell death through the MMR system. Carcinogenesis 27: 682-692.

9. Branch P, Aquilina G, Bignami M, Karran P (1993) Defective mismatch binding and a mutator phenotype in cells tolerant of DNA damage. Nature 362: 652654 .

10. Vlassenbroeck I, Califice S, Diserens AC, Migliavacca E, Straub J, et al (2008) Validation of real-time methylation-specific PCR to determine O6-
methylguanine-DNA methyltransferase gene promoter methylation in glioma J Mol Diagn 10: 332-337.

11. Berg KD, Glaser CL, Thompson RE, Hamilton SR, Griffin CA, et al. (2000) Detection of microsatellite instability by fluorescence multiplex polymerase chain reaction. J Mol Diagn 2: 20-28.

12. Esteller M. Corn PG, Baylin SB, Herman JG (2001) A gene hypermethylation profile of human cancer. Cancer Res 61: 3225-3229.

13. Hoffmann AC, Kaifi JT, Vallbohmer D, Yekebas E, Grimminger P, et al. (2009) Lack of prognostic significance of serum DNA methylation of DAPK, MGMT and GSTPI in patients with non-small cell lung cancer. J Surg Oncol 100: 414-417.

14. Scartozzi M, Franciosi V, Campanini N, Benedetti G, Barbieri F, et al. (2006) Mismatch repair system (MMR) status correlates with response and survival in Non-small cell lung cancer (NSCLC) Patients. Lung Cancer 53: 103-109.

15. Liu L, Gerson SL (2006) Targeted modulation of MGMT: clinical implications. Clin Cancer Res 12: 328-331.

16. Martinez R, Schackert HK, Appelt H, Plaschke J, Baretton G, et al. (2005) Low level microsatellite instability phenotype in sporadic glioblastoma multiforme. J Cancer Res Clin Oncol 131: 87-93.

17. Bellizi AM, Frankel WL (2009) Colorectal cancer due to deficiency in DNA mismatch repair function: a review. Adv Anat Pathol 16: 405-417.

18. Antonadou D, Paraskervaidis M, Sarris G, Coliarakis N, Economou I, et al. (2002) Phase II randomized trial of temozolomide and concurrent radiotherapy in patients with brain metastasis. J Clin Oncol 20: 3644-3650.

19. Verger E, Gil M, Yaya R, Vinolas N, Villa S, et al. (2005) Temozolomide and concomitant whole brain radiotherapy in patients with brain metastases: a phase II randomized trial. Int J Radiat Oncol Biol Phys 61: 185-191. 